\title{
What High Pressure Studies Have Taught Us About High-Temperature Superconductivity
}

\author{
JAMES S. SCHILLING \\ Department of Physics, Washington University, C.B. 1105, One Brookings Dr., St. \\ Louis, MO 63130
}

\begin{abstract}
Superconductivity is an important area of modern research which has benefited enormously from experiments under high pressure conditions. The focus of this paper will be on three classes of high-temperature superconductors: (1) the new binary compound $\mathrm{MgB}_{2},(2)$ the alkali-doped fullerenes, and (3) the cuprate oxides. We will discuss results from experiment and theory which illustrate the kinds of vital information the high-pressure variable can give to help better understand these fascinating materials.
\end{abstract}

\section{INTRODUCTION}

Superconductivity was discovered in 1911 by G. Holst and Kamerlingh Onnes in Leiden when elemental $\mathrm{Hg}$ was cooled to temperatures below $4.15 \mathrm{~K}$ [1]. Fourteen years later the same group carried out the first high-pressure experiments on a superconductor [2]. As reported by D. H. Bowen in his review paper [3]: "The first experiments in which stresses were deliberately applied to superconductors were by Sizoo and Onnes in 1925 who found that the transition temperatures of tin and indium were increased by uniaxial tension and decreased by applying pressure to the helium bath in which they were immersed". In the 76 years since these first experiments, high-pressure experiments have had a considerable impact on the field of superconductivity. This is not surprising, since the application of high pressures has:

1. led to the discovery of many new superconductors, including 22 elemental solids (B, O, Si, P, S, Ca, Sc, Fe, Ge, As, Se, Br, Sr, Y, Sb, Te, I, Cs, Ba, Bi, Ce, Lu) bringing the total number of elemental-solid superconductors to 51 .

2. aided in the synthesis of novel high quality superconducting materials. This the subject of a paper by C.W. Chu at this conference and will not be discussed here.

3. guided efforts to enhance the transition temperature $T_{c}$ through chemical means. Even without a detailed understanding of $w h y T_{c}$ changes with pressure, a large 
magnitude of the pressure derivative $d T_{c} / d P$ is a good indication that higher values of $T_{c}$ are possible at ambient pressure through chemical means. The observation of a large enhancement in $T_{c}$ under pressure in the high-temperature oxide superconductor La-Ba-Cu-O prompted the substitution of the smaller ion $\mathrm{Y}^{3+}$ for $\mathrm{La}^{3+}$ to generate lattice pressure, leading to the important discovery [4] of superconductivity in $\mathrm{YBa}_{2} \mathrm{Cu}_{3} \mathrm{O}_{7-\delta}(\mathrm{Y}-123)$ at $92 \mathrm{~K}$. Below we will see that efforts are being made to expand the lattice of hole-doped $\mathrm{C}_{60}$ in hopes of raising its $T_{c} \simeq 52 \mathrm{~K}$ to even higher values [5].

4. yielded the dependence of $T_{c}$ on sample volume and lattice parameters which helped identify the pairing mechanism and test theoretical models.

This paper will focus its attention on this fourth "benefit" of high pressure research. The types of superconductor that we consider are the binary compound $\mathrm{MgB}_{2}$, electron- and hole-doped fullerenes, and the cuprate oxides. These materials owe their extraordinary superconducting properties to their reduced (2D) dimensionality. The electron pairing leading to superconductivity takes place primarily within the $\mathrm{B}_{2}$-layers in $\mathrm{MgB}_{2}$, within the $\mathrm{CuO}_{2}$-planes in the oxides, and on the surface (2D!) of the large $\mathrm{C}_{60}$ molecule in the doped fullerenes.

The results of high pressure experiments on these important materials can be best understood if we first consider analogous experiments on conventional simple metal and transition metal superconductors.

\section{SUPERCONDUCTIVITY IN CONVENTIONAL SU- PERCONDUCTORS}

The fact that high pressure creates 22 new elemental superconductors does not imply that superconductivity is normally enhanced under pressure; in fact, just the opposite is true. The majority of the above "high-pressure superconductors" entered this state following a pressure-induced insulator $\rightarrow$ metal transition. For most known superconductors, $T_{c}$ decreases under pressure, sometimes quite rapidly; a positive value of $d T_{c} / d P$ is rather infrequent. The reason for this can be most easily understood by considering the simple metal superconductors, like $\mathrm{Sn}, \mathrm{In}, \mathrm{Pb}$, and $\mathrm{Al}$, where the conduction electrons possess $s, p$ character. In all simple metal superconductors, $d T_{c} / d P$ is negative [6]: for example, $\mathrm{Sn}(-0.482 \mathrm{~K} / \mathrm{GPa})$, In $(-0.381 \mathrm{~K} / \mathrm{GPa})$, and $\mathrm{Pb}(-0.365 \mathrm{~K} / \mathrm{GPa})$. This ubiquitous decrease in $T_{c}$ is not an electronic effect, but arises predominantly from a stiffening of the lattice with increasing pressure, the same reason the equation-of-state $V(P)$ has an upward (positive) curvature!

We can make these arguments more specific by considering the BCS expression for the transition temperature

$$
T_{c} \simeq\langle\omega\rangle \exp \left\{\frac{-1}{N\left(E_{f}\right) \mathcal{V}}\right\}
$$


where $\langle\omega\rangle$ is an average lattice-vibration frequency, $N\left(E_{f}\right)$ is the electronic density of states at the Fermi energy, and $\mathcal{V}$ is the attractive pairing interaction. Since the $s, p$ electrons in simple metals are nearly free, one expects approximately $N\left(E_{f}\right) \propto V^{+2 / 3}$ so that $N\left(E_{f}\right)$ decreases even more slowly than the sample volume $V$ with increasing pressure. However, the principal reason for the observed decrease in $T_{c}$ with pressure is that the pairing interaction $\mathcal{V}$ itself decreases by a sizeable amount due to lattice stiffening, which makes it increasingly difficult for the anions in the crystal lattice to couple with the electrons.

To put this discussion on a more quantitative basis, we need to consider the McMillan equation [7]

$$
T_{c} \simeq \frac{\langle\omega\rangle}{1.20} \exp \left\{\frac{-1.04(1+\lambda)}{\lambda-\mu^{*}(1+0.62 \lambda)}\right\},
$$

which connects the value of $T_{c}$ with fundamental parameters such as the electronphonon coupling parameter $\lambda$, an average phonon frequency $\langle\omega\rangle$, and the Coulomb repulsion which we set equal to $\mu^{*}=0.1$. The coupling parameter itself is defined by $\lambda=N\left(E_{f}\right)\left\langle I^{2}\right\rangle /\left[M\left\langle\omega^{2}\right\rangle\right]$, where $\left\langle I^{2}\right\rangle$ is the average squared electronic matrix element, $M$ the molecular mass, and $\left\langle\omega^{2}\right\rangle$ the average squared phonon frequency. Taking the logarithmic volume derivative of $T_{c}$ in Eq. (2), we obtain the simple relation

$$
\frac{d \ln T_{c}}{d \ln V}=-B \frac{d \ln T_{c}}{d P}=-\gamma+\Delta\left\{\frac{d \ln \eta}{d \ln V}+2 \gamma\right\}
$$

where $B$ is the bulk modulus, $\gamma \equiv-d \ln \langle\omega\rangle / d \ln V$ the Grüneisen parameter, $\eta \equiv$ $N\left(E_{f}\right)\left\langle I^{2}\right\rangle[8]$, and $\Delta \equiv 1.04 \lambda\left[1+0.38 \mu^{*}\right]\left[\lambda-\mu^{*}(1+0.62 \lambda)\right]^{-2}$. Eq. (3) has a simple interpretation. The first term on the right, which comes from the prefactor to the exponent in the above McMillan expression for $T_{c}$, is usually small relative to the second term. The sign of the pressure derivative $d T_{c} / d P$, therefore, is determined by the relative magnitude of the two terms in the curly brackets.

The first "electronic" term in the curly brackets involves the derivative of the Hopfield parameter $\eta \equiv N\left(E_{f}\right)\left\langle I^{2}\right\rangle$, an "atomic" property which can be calculated directly in band-structure theory. In his landmark paper [7], McMillan demonstrated that whereas individually $N\left(E_{f}\right)$ and $\left\langle I^{2}\right\rangle$ may fluctuate appreciably, their product $\eta \equiv N\left(E_{f}\right)\left\langle I^{2}\right\rangle$ changes only gradually, i.e. $\eta$ is a well behaved "atomic" property. One would thus anticipate that $\eta$ changes in a relatively well defined manner under pressure, reflecting the character of the electrons near the Fermi energy. An examination of the body of high-pressure data on simple $s, p$-metal superconductors, in fact, reveals that $\eta$ normally increases under pressure at a rate close to $d \ln \eta / d \ln V \approx-1$ [9]. For transition-metal (d-electron) superconductors, on the other hand, Hopfield has pointed out [8] that the larger value $d \ln \eta / d \ln V \approx-3$ to -4 is more appropriate.

Let us now apply Eq. (3) to an analysis of $d T_{c} / d P$ for simple metal superconductors. The second "lattice" term in the curly brackets in Eq. (3) is positive since the 
lattice term is positive $(2 \gamma \approx+3$ to +5$)$ and dominates over the negative electronic term $d \ln \eta / d \ln V \approx-1$. Since $\Delta$ is always positive and $-\gamma$ is relatively small, the sign of $d T_{c} / d P$ is negative, opposite to that in the curly brackets. This accounts for the universal decrease of $T_{c}$ with pressure due to lattice stiffening in simple metals. In $\mathrm{Sn}$, for example, $T_{c}$ decreases under pressure at the rate $d T_{c} / d P \simeq-0.482 \mathrm{~K} / \mathrm{GPa}$ which leads to $d \ln T_{c} / d \ln V \simeq+7.2$ [6]. Inserting for $\operatorname{Sn} T_{c 0} \simeq 3.73 \mathrm{~K},\langle\omega\rangle \simeq 110$ $\mathrm{K}$ [10], and $\mu^{*}=0.1$ into the above McMillan equation, we obtain $\lambda \simeq 0.69$ from which follows that $\Delta \simeq 2.47$. Inserting the above values into Eq. (3) and setting $d \ln \eta / d \ln V \approx-1$, we can solve Eq. (3) for the Grüneisen parameter to obtain $\gamma \simeq+2.46$, in reasonable agreement with the experimental value $\gamma \approx+2.1$ [6]. Similar results are obtained for other conventional simple metal BCS superconductors.

In transition metal superconductors the electrons taking part in the superconductivity have predominantly $d$ character which often leads to higher values of the density of states $N\left(E_{f}\right)$ and transition temperature $T_{c}$. In many transition metals $T_{c}$ decreases with pressure, but in some $T_{c}$ increases. Indeed, under pressure $N\left(E_{f}\right)$ can either decrease or increase; should $E_{f}$ lie on the low energy side of a peak in $N(E)$, $s \rightarrow d$ electron transfer under pressure would lead to an increase in $N\left(E_{f}\right)$, and vice versa should $E_{f}$ lie on the high energy side of a peak. The moderating influence of the change in $\left\langle I^{2}\right\rangle$ under pressure leads to the universal increase of their product $\eta \equiv N\left(E_{f}\right)\left\langle I^{2}\right\rangle$ according to $d \ln \eta / d \ln V \approx-3$ to -4 , as pointed out by Hopfield [8]. If this relatively large electronic term becomes larger than the lattice term $2 \gamma$ in Eq. (3), $T_{c}$ would be expected to increase with pressure; this is, in fact, observed in the transition metals $\mathrm{V}$ [1] and La [12], among others. Unlike for $s, p$ metals, the pressure dependence $T_{c}(P)$ for transition metals follows no universal behavior,

reflecting the additional complexity, and potency, of the electronic properties in a $d$ electron system.

\section{SUPERCONDUCTIVITY IN $\mathrm{MgB}_{2}$}

The discovery of superconductivity at the high temperature $T_{c} \approx 40 \mathrm{~K}$ in the simple $s, p$-metal compound $\mathrm{MgB}_{2}$ was quite unexpected [13]. The absence [14] of the problematic weak-link behavior of the high- $T_{c}$ oxides and the relative ease of synthesis in various forms [15 has raised hopes that $\mathrm{MgB}_{2}$ may be suitable for numerous technological applications. Efforts to enhance the value of $T_{c}$ in this class of superconductor would be aided by the identification of the superconducting mechanism and by establishing systematics in the superconducting and normal-state properties. $\mathrm{MgB}_{2}$ is a quasi-2D material with strong covalent bonding within the boron layers. It is thus not surprising that the compression is anisotropic [16, 17, 27, 18], the most accurate structural measurements [27] revealing that under hydrostatic pressure the initial compression along the $c$ axis is $64 \%$ greater than along the $a$ axis; the bulk modulus is $B=147.2(7)$. The anisotropy in the superconducting properties is also appreciable, the upper critical field ratio $H_{c 2}^{a b} / H_{c 2}^{c}$ reportedly being $2-3$ [19, 20], less 
than that observed in the high- $T_{c}$ oxides [21]. A full characterization of the remaining anisotropic properties awaits the synthesis of sufficiently large single crystals.

Soon after the discovery of superconductivity in $\mathrm{MgB}_{2}$, three groups [22, 23, 24] reported that $T_{c}$ decreased under high pressure, but the rate of decrease varied considerably from $-1.6 \mathrm{~K} / \mathrm{GPa}$ [22] to $-1.9 \mathrm{~K} / \mathrm{GPa}$ [23] in piston-cylinder studies with Fluorinert pressure medium, to $\sim-0.6 \mathrm{~K} / \mathrm{GPa}$ in quasi-hydrostatic studies [24] to 25 GPa with solid steatite pressure medium. Choi et al. [25] have recently carried out resistivity studies to $1.5 \mathrm{GPa}$ pressure in daphne-kerosene pressure medium, obtaining $d T_{c} / d P \simeq-1.36 \mathrm{~K} / \mathrm{GPa}$. The differing pressure dependences may be due to differences in the samples and/or to shear stress effects in the frozen or solid pressure media.

We recently carried out He-gas hydrostatic pressure experiments [26] on the same high quality isotopically pure $\left({ }^{11} \mathrm{~B}\right)$ sample used in the above structural studies [27]. For $P \lesssim 0.5 \mathrm{GPa}$ helium is fluid at $T_{c} \approx 39 \mathrm{~K}$. At higher pressures the shear stresses are held to a minimum by the carefully controlled manner [28] in which solid helium is allowed to freeze around the sample. The dependence of $T_{c}$ on hydrostatic pressure is seen in Fig. 1(a) to be highly linear, $d T_{c} / d P \simeq-1.11(2)$ $\mathrm{K} / \mathrm{GPa}$ (yielding $d \ln T_{c} / d \ln V=B d \ln T_{c} / d P \simeq+4.16$ ), and does not depend on the pressure/temperature history of the sample. Such history effects are rare in superconductors without pressure-induced phase transitions, but do occur in certain high- $T_{c}$ oxides containing defects with appreciable mobility at RT [29. In addition, we observed that $d T_{c} / d P$ remained unchanged if neon was substituted for He as pressure medium, confirming the absence of intercalation effects in $\mathrm{MgB}_{2}$. Lorenz et al. [30] have very recently carried out He-gas studies to $0.8 \mathrm{GPa}$ on two $\mathrm{MgB}_{2}$ samples with differing $T_{c 0}$ values $39.2 \mathrm{~K}$ and $37.5 \mathrm{~K}$ obtaining $d T_{c} / d P \simeq-1.07$ $\mathrm{K} / \mathrm{GPa}$ and $-1.45 \mathrm{~K} / \mathrm{GPa}$, respectively. These authors conclude that differences in the samples themselves, and not shear stress effects, are responsible for the differing $d T_{c} / d P$ values.

In Fig. 1(b) we show the dependence of $T_{c}$ on pressure to $20 \mathrm{GPa}$ for $\mathrm{MgB}_{2}$ using a diamond-anvil-cell (DAC) with dense helium pressure medium [31], thus extending the pressure range of the above He-gas studies nearly thirtyfold. $T_{c}$ is seen to decrease approximately linearly with pressure to $10 \mathrm{GPa}$, consistent with the rate -1.11 K/GPa (dashed line). Very recently Tissen et al. 32 have carried out ac susceptibility measurements in a DAC to $28 \mathrm{GPa}$ on a $\mathrm{MgB}_{2}$ sample with $T_{c 0} \simeq 37.3$ $\mathrm{K}$ at ambient pressure. They find the high initial slope $d T_{c} / d P \simeq-2 \mathrm{~K} / \mathrm{GPa}, T_{c}$ decreasing to $11 \mathrm{~K}$ at $20 \mathrm{GPa}$ and $6 \mathrm{~K}$ at $28 \mathrm{GPa}$. They also report that the pressure dependence $T_{c}(P)$ shows a bump near $9 \mathrm{GPa}$ which they speculate may arise from an electronic Lifshitz transition. Further experiments are necessary to determine whether this bump is intrinsic to the sample or the result of shear stress effects in the frozen methanol-ethanol pressure medium. 
Fig. 1. (a)(top) Superconducting transition temperature onset versus applied pressure from Ref. [26]. Numbers give order of measurement. A typical error bar for $T_{c}( \pm 0.01 \mathrm{~K})$ is given in lower left corner; the error in pressure is less than the symbol size. Pressure was either changed at RT (unprimed numbers) or at low temperatures $\sim 60 \mathrm{~K}$ (primed numbers). (b)(bottom) Superconducting transition temperature midpoint versus pressure to 20 GPa from diamond-anvil-cell measurements in Ref. [31]. Data taken first for monotonically increasing pressure $(\bullet)$, then for monotonically decreasing pressure (o). The straight dashed line has slope $-1.11 \mathrm{~K} / \mathrm{GPa}$.

We now apply the same analysis carried out above for simple metal superconductors to $\mathrm{MgB}_{2}$ to see whether $T_{c}(P)$ from our measurements is consistent or not with BCS electron-phonon coupling theory. We first analyze the He-gas data which gives the initial dependence. Using the average phonon energy from inelastic neutron studies [33] $\langle\omega\rangle=670 \mathrm{~K}, T_{c 0} \simeq 39.25 \mathrm{~K}$, and $\mu^{*}=0.1$, we obtain from the above relations $\lambda \simeq 0.90$ and $\Delta \simeq 1.75$. Our estimate of $\lambda \simeq 0.90$ agrees well 
with those of other authors [35, 34]. Since the pairing electrons in $\mathrm{MgB}_{2}$ are believed to be $s, p$ in character [37, 36, 35, 38], we set $d \ln \eta / d \ln V \approx-1$, a value close to $d \ln \eta / d \ln V=B d \ln \eta / d P \approx-0.81$, where $B=147.2 \mathrm{GPa}$ from Ref. [27] and $d \ln \eta / d P \approx+0.55 \% / \mathrm{GPa}$ from first-principles electronic structure calculations by Medvedera et al. [39]. Inserting the above values of $d \ln T_{c} / d \ln V=+4.16, \Delta=1.75$, and $d \ln \eta / d \ln V=-1$ into Eq. (3), we find $\gamma=2.36$, in reasonable agreement with the value $\gamma \approx 2.9$ from Raman spectroscopy studies 18 or $\gamma \approx 2.3$ from ab initio electronic structure calculations on $\mathrm{MgB}_{2}$ 40. The He-gas $T_{c}(P)$ data are thus clearly consistent with electron-phonon pairing in $\mathrm{MgB}_{2}$.

A more stringent test of this conclusion is provided by the DAC $T_{c}(P)$ data in Fig. 1(b) which cover a relatively large $\sim 10 \%$ change in volume. We would like to see whether we can reproduce the DAC data using the McMillan equation and suitably extrapolating the parameters used in the above analysis of the He-gas data. As pointed out by Chen et al. [41], an appropriate method of extrapolation is to integrate the volume derivatives of the above parameters $\gamma \equiv-d \ln \langle\omega\rangle / d \ln V=$ +2.36 and $d \ln \lambda / d \ln V=d \ln \eta / d \ln V-d \ln \left\langle\omega^{2}\right\rangle / d \ln V=-1-2(-2.36)=+3.72$ to obtain $\langle\omega\rangle=(670 \mathrm{~K})\left(V / V_{0}\right)^{-2.36}$ and $\lambda=0.90\left(V / V_{0}\right)^{3.72}$. Inserting these two volume dependences in the McMillan equation, and assuming $\mu^{*}=0.1$ is independent of pressure [41], we obtain the dependence of $T_{c}$ on relative volume $V / V_{0}$. This can be converted to the dependence of $T_{c}$ on pressure $P$ by using the Murnaghan equation-ofstate $V(P) / V_{0}=\left[1+B^{\prime} P / B\right]^{-1 / B^{\prime}}$ where we use the value $B=147.2 \mathrm{GPa}$ from Ref. 27] and the canonical value $B^{\prime} \equiv d B / d P=4$ supported by a recent calculation [42]. As seen in Fig. 1(b), the agreement of this calculated $T_{c}(P)$ dependence (solid line) with the experimental data is quite impressive. According to this estimate, a pressure of $P \approx 50 \mathrm{GPa}$ would be required to drive $T_{c}$ to below $4 \mathrm{~K}$. A similar calculation was very recently carried out by Chen et al. 41] over a much wider pressure range; this paper also contains a detailed discussion of the pressures dependences of $\eta, \lambda$, and $\mu^{*}$.

The good agreement between the experimental data to $20 \mathrm{GPa}$ and the predictions of the McMillan formula using the volume dependences determined from the He-gas high-pressure data to 0.7 GPa provides substantial evidence that superconductivity in $\mathrm{MgB}_{2}$ originates from standard BCS phonon-mediated electron pairing. This finding agrees with high precision isotope effect experiments [43, 44], among others. The fact that the $\mathrm{B}$ isotope effect is fifteen times that for $\mathrm{Mg}$ [44] is clear evidence that the superconducting pairing originates within the graphite-like $\mathrm{B}_{2}$-layers

\section{SUPERCONDUCTIVITY IN THE ALKALI-DOPED FULLERENES}

Another class of superconductors having very high values of $T_{c}$ are the alkali-doped fullerides $\mathrm{A}_{3} \mathrm{C}_{60}$, where $\mathrm{A}=\mathrm{K}, \mathrm{Rb}, \mathrm{Cs}$, or some combination thereof 45. As for the simple metal and $\mathrm{MgB}_{2}$ superconductors, $T_{c}$ for the alkali-doped fullerides is found to 
decrease under the application of hydrostatic pressure [46]. For example, for $\mathrm{Rb}_{3} \mathrm{C}_{60}$, where $T_{c 0} \simeq 29.5 \mathrm{~K}$, we obtained $d T_{c} / d P \simeq-8.7 \mathrm{~K} / \mathrm{GPa} 47$. From our measurement of the bulk modulus in a neutron diffraction experiment [48], $B=18.3 \mathrm{GPa}$, we estimated $d \ln T_{c} / d \ln V=B d \ln T_{c} / d P \simeq+5.4$, a value intermediate between that for $\mathrm{MgB}_{2}(+4.16)$ and that for $\mathrm{Sn}(+7.2)$. One is tempted to account for the decrease in $T_{c}$ with pressure for $\mathrm{Rb}_{3} \mathrm{C}_{60}$ or the other $\mathrm{A}_{3} \mathrm{C}_{60}$ fullerides by invoking pressure-induced lattice stiffening. Such an attempt, however, fails. In contrast to $\mathrm{MgB}_{2}$ and $\mathrm{Sn}$, the electronic bandwidth is quite narrow, leading to the expectation of a sizeable decrease in $N\left(E_{f}\right)$ under pressure. To determine the reason for the large negative value of $d T_{c} / d P$, we measured in a single experiment the pressure dependence of both $T_{c}$ and $N\left(E_{f}\right)$ for $\mathrm{Rb}_{3} \mathrm{C}_{60}$, determining in the process the functional dependence of $T_{c}$ on $N\left(E_{f}\right)$ [47]. A detailed analysis revealed that weak-coupling theory can account for these pressure dependences as long as the characteristic energy of the intermediary boson is between $300 \mathrm{~K}$ and $800 \mathrm{~K}$, typical energies for the high frequency on-ball phonons. The mechanism behind the large negative value of $d T_{c} / d P$ in $\mathrm{Rb}_{3} \mathrm{C}_{60}$ is thus not lattice stiffening, as in the simple metals and $\mathrm{MgB}_{2}$, but a sharp decrease in the electronic density of states $N\left(E_{f}\right)$ with pressure.

This result sheds light on the observed increase in $T_{c}$ in $\mathrm{A}_{3} \mathrm{C}_{60}$ as the larger $\mathrm{Rb}^{+1}$ ion is substituted for the smaller $\mathrm{K}^{+1}$, thus expanding the lattice, as seen in Fig. 2. In fact, it was widely believed that the relationship between $T_{c}$ and the lattice parameter $a$ followed a universal behavior in both cation substitution and high-pressure experiments. Our combined equation-of-state and $T_{c}(P)$ studies on $\mathrm{Rb}_{3} \mathrm{C}_{60}$, however, revealed that two different dependences are found, as seen in Fig. 2. The reason for this effect is not known, but may have to do with different rotationally ordered states of the $\mathrm{C}_{60}$ molecule in the two cases which influences the density of states and thus $T_{c}$.

Fig. 2. $\mathrm{T}_{c}$ versus lattice parameter from Ref. [48] for both $(\bullet)$ high-pressure experiments on $\mathrm{Rb}_{3} \mathrm{C}_{60}$ and (o) cation-substitution experiments. Only the cation(s) to $\mathrm{C}_{60}$ are listed in the figure. Solid line is guide to eye.

Using gate-induced doping in a field-effect transistor configuration, Schön et al. 
have demonstrated superconductivity in both electron- [49] and hole-doped [50] $\mathrm{C}_{60}$ single crystals with lattice parameter $14.16 \AA$. The maximum values of $T_{c}$ are found to be $11 \mathrm{~K}$ and $52 \mathrm{~K}$, respectively. The latter value is a record for a non-cuprate superconductor. The higher value of $T_{c}$ for hole doping compared to electron doping is apparently correlated with the higher density of states in the valence band (HOMO) compared with the conduction band (LUMO).

From the above discussion, $T_{c} \simeq 52 \mathrm{~K}$ for optimally hole-doped $\mathrm{C}_{60}$ would evidently be expected to increase further if its lattice parameter $a$ could be expanded. We found above for $\mathrm{Rb}_{3} \mathrm{C}_{60}$ that $d \ln T_{c} / d \ln V=d \ln T_{c} / 3 d \ln a \simeq+5.4$, which implies that $d \ln T_{c} / d \ln a \simeq+16.2$. This means that one would expect $T_{c}$ to increase about 16 times faster than the lattice parameter $a$. If we expand the lattice parameter $a$ of $\mathrm{C}_{60}$ by $2 \%$, so that it equals that of $\mathrm{Rb}_{3} \mathrm{C}_{60}$, one would expect $T_{c}$ to increase by approximately $16.2 \times 2 \% \simeq 32 \%$, implying an increase in $T_{c}$ from $52 \mathrm{~K}$ to nearly 70 $\mathrm{K}$. To test the above, it would be of interest to expand $\mathrm{C}_{60}$ 's lattice, perhaps through suitable intercalation of rare gas atoms.

\section{SUPERCONDUCTIVITY IN THE CUPRATE OXIDES}

It has been nearly 15 years since the discovery [51] of high- $T_{c}$ superconductivity in the cuprate oxide La-Ba-Cu-O at $T_{c} \approx 35 \mathrm{~K}$. In spite of enormous efforts since then, including over 50,000 experimental and 20,000 theoretical papers, there is still no consensus on the underlying mechanism responsible for the superconducting pairing. This is one of the great unsolved problems in Condensed Matter Physics. The maximum value of $T_{c}$ has improved from initially $\sim 35 \mathrm{~K}$ for La-Ba-Cu-O and related systems to $134 \mathrm{~K}$ for $\mathrm{HgBa}_{2} \mathrm{Ca}_{2} \mathrm{Cu}_{3} \mathrm{O}_{8+\delta}(\mathrm{Hg}-1223)$ at ambient pressure in 1993 [52 and $\sim 160 \mathrm{~K}$ for the same compound at $30 \mathrm{GPa}$ pressure in 1994 53. There have even been unconfirmed reports of superconductivity at $250 \mathrm{~K}$ in $\mathrm{Tl}_{2} \mathrm{Ba}_{2} \mathrm{Ca}_{2} \mathrm{Cu}_{3} \mathrm{O}_{10+\delta}$ (Tl-2223) above $4 \mathrm{GPa}$ [54] and at $330 \mathrm{~K}$ in the $\mathrm{Pb}-\mathrm{Ag}-\mathrm{C}-\mathrm{O}$ system at ambient pressure [55].

Unfortunately, Nature has been particularly inventive in her efforts to thwart understanding. The distorted modified perovskite structure of these materials undergoes subtle structural changes when temperature or pressure is varied which complicates the interpretation of experimental data. Defects of many different kinds have a profound influence on the superconducting properties, including the value of $T_{c}$. An example is the emergence of the low-temperature-tetragonal (LTT) phase in $\mathrm{La}_{2-x} \mathrm{Ba}_{x} \mathrm{CuO}_{4}$ for $x \approx 0.125$ which completely suppresses $T_{c}$ from its nominal value of $\sim 37 \mathrm{~K}$. Because of the strong inverse parabolic dependence of $T_{c}$ on the carrier concentration $n$ seen in Fig. 3 [56], small changes in $n$ due to the influence of defects can have a surprising large effect on $T_{c}$.

In spite of the complexity of these materials, researchers [57] have been able to identify a number of important guidelines for enhancing the value of $T_{c}$ in the superconducting oxides: (1) the carrier concentration $n$ in the $\mathrm{CuO}_{2}$ planes should 
be varied through suitable cation substitution until its optimal value is reached (see Fig. 3); (2) try to increase the number of $\mathrm{CuO}_{2}$ planes which lie close together (in a packet) in the oxide structure while maintaining optimal doping - "respectable" one-plane systems, like Tl-2201, have $T_{c}$ values in the range $90-100 \mathrm{~K}$, two-plane systems in the range $100-120 \mathrm{~K}$, and three-plane systems in the range $120-140 \mathrm{~K}$; (3) try to position defects as far from the $\mathrm{CuO}_{2}$ planes as possible; and (4) since $T_{c}$ is diminished with increasing buckling angle in the $\mathrm{CuO}_{2}$ planes, develop structures where the $\mathrm{CuO}_{2}$ planes are as flat as possible.

We would like to pose the following question: are there perhaps other guidelines to maximize $T_{c}$ which high pressure studies can give us? Can high pressure experiments give us NEW information not available from other studies? The most studied superconducting property under pressure is $T_{c}(P)$ which depends on the system studied, the doping level $n$, the type and mobility of defects, and, in some cases, on the pressure medium used [9, 58]. However, considering the totality of $T_{c}(P)$ data, one feature stands out: that more often than not $T_{c}$ increases with pressure, as first recognized by Schirber [59]. The foregoing discussion should help the reader appreciate that an increase in $T_{c}$ with pressure is something special! In the case of the optimally doped $\mathrm{Hg}$ compounds with one, two and three $\mathrm{CuO}_{2}$ planes, the initial rate of increase is identical, $d T_{c} / d P \approx+1.75 \mathrm{~K} / \mathrm{GPa}$ [60]. It is this constant but perservering increase in $T_{c}$ with pressure which allows $T_{c}$ in $\mathrm{Hg}-1223$ to increase from $134 \mathrm{~K}$ at ambient pressure to $\sim 160 \mathrm{~K}$ at $30 \mathrm{GPa}$ at which pressure $T_{c}(P)$ passes through a maximum [53].

In the cuprate oxides, the canonical change in $T_{c}$ with pressure is that it first increases with pressure, passes through a maximum value at some critical

Fig. 3. Inverted parabolic dependence of $\mathrm{T}_{c}$ on hole-carrier content for the superconducting oxides adapted from Ref. [56]. Representative experimental values of $\mathrm{dT} c / \mathrm{dP}$ for underdoped, optimally doped and overdoped sampes are given. 
pressure, and then decreases [9, 58]. What causes this $T_{c}(P)$ dependence? An examination of Fig. 3 leads one to the idea that perhaps the hole-carrier concentration $n$ increases with pressure so that for an underdoped sample, where $n<n_{\text {opt }}, T_{c}(P)$ simply tracks the canonical bell-shaped $T_{c}(n)$ curve. Indeed, Hall effect studies show that $n$ normally increases with pressure at the rate $+10 \% / \mathrm{GPa}$ [9]. If this were the whole story, then for an optimally doped sample one would expect $d T_{c} / d P=$ $\left(d T_{c} / d n\right)(d n / d P)=0$, since $T_{c}(n)$ is at an extremum for $n=n_{\text {opt }}$ (see Fig. 3). As indicated in Fig. 3, however, this expectation from this simple charge-transfer model is not confirmed in experiment. For "normal" high- $T_{c}$ oxides, $d T_{c} / d P \approx+1$ to +2 at optimal doping! This means that there are at least two effects determining the total pressure dependence of $T_{c}$

$$
\frac{d T_{c}}{d P}=\left(\frac{d T_{c}}{d P}\right)^{\text {intrinsic }}+\left(\frac{d T_{c}}{d n}\right)\left(\frac{d n}{d P}\right),
$$

an intrinsic dependence $\left(d T_{c} / d P\right)^{\text {intrinsic }} \approx+1$ to +2 , and a second dependence arising from pressure-induced changes in the carrier concentration $n$ in the $\mathrm{CuO}_{2}$ planes. It is this intrinsic term which promises to tell us something new about high- $T_{c}$ superconductors, something we perhaps could not have learned from ambient pressure experiments under varying sample stoichiometries and structure types. However, we have only evaluated $\left(d T_{c} / d P\right)^{\text {intrinsic }}$ at optimal doping. It is certainly possible that this intrinsic dependence varies with the carrier concentration. To extract the intrinsic term from $T_{c}(P)$ data with varying values of $n$, we must be able to estimate the second term on the right in Eq. (4), the charge transfer term. This is no mean feat since this term depends on both the rate of change of $n$ with pressure, as well as $d T_{c} / d n$.

There are, however, further problems which make the extraction of the intrinsic term even more difficult. In many superconducting oxides the application of pressure doesn't simply compress the lattice, but also prompts mobile oxygen defects to assume a greater degree of local order [9, 61]. This leads to relaxation effects which are both temperature and pressure dependent. The oxygen chain sublattice in $\mathrm{YBa}_{2} \mathrm{Cu}_{3} \mathrm{O}_{7-y}$ (Y-123), for example, is partially occupied with oxygen anions with a considerable mobility at room temperature. This allows these oxygen defects to assume a myriad of different substructures or local ordered states which can influence the value of $T_{c}$. Under pressure the oxygen defects may migrate from one local ordered state to another, but at a progressively slower rate, the higher the pressure [62]. In fact, one can measure the pressure dependence of the relaxation time $\tau(P)$ and use this to estimate the most likely diffusion path of oxygen defects through the solid 63. For studies of the $T_{c}(P)^{\text {intrinsic }}$, however, these relaxation effects are more than just a nuisance. One strategy to eliminate the relaxation effects completely is to carry out the entire high pressure experiment at low temperatures, allowing $\tau$, which depends exponentially on temperature, to become extremely large. The hope would be that the elimination of relaxation effects would turn the complex dependency $T_{c}(n, P)$ into 
a simple one. Unforunately, experiments to $20 \mathrm{GPa}$ in a DAC on Y-123 carried out solely at temperatures low enough $(<90 \mathrm{~K})$ to suppress oxygen ordering brought little simplification [62]. In the Y-123 system, at least, the extraction of the dependence $T_{c}(P)^{\text {intrinsic }}$ would seem out of reach.

Fortunately, these relaxation effects operate by changing the carrier concentration $n$. By considering pressure derivatives $d T_{c} / d P$ only for optimally doped samples, we can eliminate not only any changes in $T_{c}$ from the normal increase in $n$ with pressure, but also those changes arising from oxygen ordering phenomena. Perhaps the most important fundamental result from all high pressure experiments on the optimally doped oxides is that $d T_{c} / d P=\left(d T_{c} / d P\right)^{\text {intrinsic }} \approx+1.5 \mathrm{~K} / \mathrm{GPa}$ which corresponds to the volume dependence $T_{c} \propto V^{-1.2}$ or

$d \ln T_{c} / d \ln V \simeq-1.2$ [9], a far weaker volume dependence than obtained for the $s, p$ metal systems $\mathrm{Sn}(+7.2)$ or $\mathrm{MgB}_{2}(+4.16)$ where $T_{c}$ increases with pressure. This comparison emphasizes how small the percent change in $T_{c}$ under pressure really is for the superconducting oxides. The fact that numerous different systems yield approximately the same volume dependence speaks against an increase in the density of states $N\left(E_{f}\right)$ as being responsible for the intrinsic $T_{c}$ enhancement under pressure. In fact, early studies by us of the spin susceptibility of $\mathrm{La}_{1.85} \mathrm{Sr}_{0.15} \mathrm{CuO}_{4}$ 64 and $\mathrm{Y}-123$ 65 could detect no change whatsoever in $N\left(E_{f}\right)$ under pressure. From Eq. (1) it thus follows that the increase in $T_{c}$ under pressure must come from an increase in the coupling strength $\mathcal{V}$ itself.

To go further in the analysis it is necessary to ascertain what structural feature in the highly anisotropic oxides is responsible for the increase in $\mathcal{V}$ under pressure. Uniaxial pressure experiments provide information on the changes occurring in $T_{c}$ for uniaxial stress applied both parallel and perpendicular to the $\mathrm{CuO}_{2}$ planes. One of the most important experiments in this regard was carried out in 1997 by Meingast et al. [66] on Ca-substituted Y-123. These studies revealed that the compression along the $c$ axis (perpendicular to the $\mathrm{CuO}_{2}$ planes) has no effect on $T_{c}$, whereas compression in the $\mathrm{CuO}_{2}$ planes caused the ubiquitous increase in $T_{c}$. A wealth of further uniaxial pressure experiments support this conclusion. This prompted Wijngaarden et al. 67] to state in 1999 that "Hence, there is quite some evidence that $\Delta c$ mainly influences doping, while $\Delta a$ mainly influences the intrinsic $T_{c}$ ". If we now use the available anisotropy compressibility data to convert the volume dependence $T_{c} \propto V^{-1.2}$ into a dependence on the in-plane lattice parameter $a$, we obtain

$$
T_{c} \propto a^{-4.5}
$$

so that $T_{c}$ is approximately proportional to the inverse square of the area of the $\mathrm{CuO}_{2}$ planes. This is perhaps the single most important fundamental result of all high pressure experiments on the cuprate oxides. The message is: to further enhance $T_{c}$, try to find structures which are capable of compressing the $\mathrm{CuO}_{2}$ planes without adding defects or increasing the buckling of these planes. Obviously this may not be easy to accomplish since these planes are quite stiff and it would be anticipated that 
structural attempts to compress the planes could easily cause them to buckle.

The next question is whether there is any correlation between the value of $T_{c}$ at ambient pressure and the value of the in-plane lattice parameter $a$. In Fig. 4 it is seen that there is no such correlation. The single-plane material with the highest value of $T_{c}(98 \mathrm{~K})$ is $\mathrm{Hg}-1201$ which has the largest value of $a$. In addition, the compound in Fig. 4 with the lowest value of $T_{c}$ is $\mathrm{La}_{1.85} \mathrm{Sr}_{0.15} \mathrm{CuO}_{4}$ which has the smallest value of $a$ ! We note that applying $4 \mathrm{GPa}$ pressure decreases $a$ by about $1 \%$ which would generate an increase in $T_{c}$ of about $6 \mathrm{~K}$. Within that range of $a$ in Fig. 4 the variation of $T_{c}$ for the compounds listed is much greater. This simply means that the compounds presently available have not yet played the high-pressure card, i.e. they haven't made use of the fact that $T_{c}$ will increase appreciably if $a$ is reduced. The system that managed to reduce $a$ the most is $\mathrm{La}_{1.85} \mathrm{Sr}_{0.15} \mathrm{CuO}_{4}$; perhaps the reason that it's $T_{c}$ value is the lowest of all systems in Fig. 4 arises from the strong structural distortions leading to considerable plane buckling.

Parameter Graphic.png

Fig. 4. Average lattice parameter in the $\mathrm{CuO}_{2}$ plane for representative superconducting oxide systems at ambient pressure.

To summarize, there are at least three principal ways that high external or lattice (chemical) pressures can be used to help maximize the value of $T_{c}$ in the superconducting oxides:

1. Use pressure to reach optimal doping in system with few defects in or near the $\mathrm{CuO}_{2}$ planes. This may be the best (only) way to reach optimal doping in systems 
with more than three $\mathrm{CuO}_{2}$ planes.

2. Use pressure to help flatten the $\mathrm{CuO}_{2}$ planes.

3. Apply pressure to reduce the area of the $\mathrm{CuO}_{2}$ planes, keeping them flat.

4. Use the result $T_{c} \propto a^{-4.5}$ to help identify the correct theoretical model and then apply this model to help further optimize the value of $T_{c}$. This will be the subject of a future paper.

\section{ACKNOWLEDGEMENTS}

The author would like the following present and past students in his group at Washington University for their excellent high-pressure work on superconducting materials: S. Deemyad, J. Diederichs, J.J. Hamlin, A.K. Klehe, S. Klotz, C.W. Looney, S. Sadewasser, and T. Tomita. Work at Washington University supported by NSF grant DMR-0101809.

\section{REFERENCES}

\section{References}

[1] Onnes, H.K. (1911), Akad. van Wetenschappen 14, 113.

[2] Sizoo, G.J. and Onnes, H.K. (1925), Commun. Phys. Lab. Univ. Leiden, No. 180b.

[3] Bowen, D.H. (1963), in R.S. Bradley (ed.), High Pressure Physics and Chemistry I, Academic Press, New York, pp. 355-373.

[4] Wu, M.K., Ashburgn, J.R., Torng, C.J., Hor, P.H., Meng, R.L., Gao, L., Huang, Z.J., Wang, Y.Q., and Chu, C.W. (1987), Phys. Rev. Lett. 58, 908-910.

[5] Schön, J.H., Kloch, Ch., and Batlogg, B. (2000), Nature 408, 549.

[6] Eiling, A., and Schilling, J.S. (1981), J. Phys. F 11, 623.

[7] McMillan, W.L. (1968), Phys. Rev. 167, 331; Allen, P.B., and Dynes, R.C. (1975), Phys. Rev. B 12, 905.

[8] Hopfield, J.J. (1971), Physica 55, 41.

[9] Schilling, J.S., and Klotz, S. (1992) in D.M. Ginsberg (ed.), Physical Properties of High Temperature Superconductors, Vol. III, World Scientific, Singapore, p. 59. 
[10] Dynes, R.C. (1972), Solid State Commun. 10, 615.

[11] Smith, T.F., and. Luo, H.L. (1967), J. Phys. Chem. Solids 28, 569.

[12] Smith, T.F. (1972), J. Phys. F 2, 946.

[13] Nagamatsu, J., Nakagawa, N., Muranaka, T., Zenitani, Y., and Akimitsu, J. (2001), Nature 410, 63.

[14] Larbalestier, D.C., Cooley, L.D., Rikel, M.O., Polyanskii, A.A., Jiang, J., Patnaik, S., Cai, X.Y., Feldmann, D.M., Gurevich, A., Squitieri, A.A., Naus, M.T., Eom, C.B., Hellstrom, Cava, R., Regan, K.A., Rogado, N., Hayward, M.A., He, T., Slusky, J.S., Khalifah, P., Inumaru, K., Haas, M. Nature 410, 186.

[15] Canfield, P.C., Finnemore, D., Bud'ko, S.L., Ostenson, J.E., Lapertot, G., Cunningham, C.E., and Petrovic, C. (2001), Phys. Rev. Lett. 86, 2423.

[16] Vogt, T., Schneider, G., Hriljac, J.A., Yang, G., Abell, J.S. (2001), Phys. Rev. B 63, $220505(\mathrm{R})$.

[17] Prassides, K., Iwasa, Y., Ito, T., Chi, D.H., Uehara, K., Nishibori, E., Takata, M., Sakata, S., Ohishi, Y., Shimomura, O., Muranaka, T., Akimitsu, J.(2001), Phys. Rev. $B$ 64, 012509 .

[18] Goncharov, A.F., Struzhkin, V.V., Gregoryanz, E., Hu, J., Hemley, R.J., Mao, Ho-k., Lapertot, G., Bud'ko, S.L., Canfield, P.C., preprint cond-mat/0104042.

[19] de Lima, O.F., Ribeiro, R.A., Avila, M.A., Cardoso, C.A., and Coelho, A.A. (2001), Phys. Rev. Lett. 86, 5974; Jung, C.U., Choi, J.-H., Chowdhury, P., Kim, K.H.P., Park, M.-S., Kim, H.-J., Kim, J. Y., Du, Z., Kim, M.-S., Kang, W.N., Lee, S.-Ik, Sund, S.H., and Lee, J.Y., preprint cond-mat/0105330.

[20] Lee, S. Mori, H., Masui, T., Eltsev, Yu., Yamamoto, A., and Tajima, S., preprint cond-mat/0105545.

[21] See, for example: Welp, U., Kwok, W.K., Crabtree, G.W., Vandervoort, K.G., and Liv, J.Z. (1989), Phys. Rev. Lett. 62, 1908.

[22] Lorenz, B., Meng, R.L., Chu, C.W. (2001), Phys. Rev. B 64, $01250 \%$.

[23] Saito, E., Taknenobu, T., Ito, T., Iwasa, Y., Prassides, K., and Arima, T. J. (2001), J. Phys.: Condens. Matter 13, L267.

[24] Monteverde, M., Núñez-Regueiro, M., Rogado, N., Regan, K.A., Hayward, M.A., He, T., Loureiro, S.M., Cava, R.J. (2001), Science 292, 75.

[25] Choi, E.S., Kang, W., Kim, J.Y., Park, M.-S., Jung, C.U., Kim, H.-J., and Lee, S.-Ik, preprint cond-mat/0104454. 
[26] Tomita, T., Hamlin, J.J., Schilling, J.S., Hinks, D.G., and Jorgensen, J.D. (2001), Phys. Rev. B 64, 092505; Schilling, J.S., Jorgensen, J.D., Hinks, D.G., Deemyad, S., Hamlin, J., Looney, C.W., and Tomita, T. (2001), in Studies of High Temperature Superconductors, Vol. 38, A.V. Narlikar (ed.), Nova Science Publishers, New York.

[27] Jorgensen, J.D., Hinks, D.G., Short, S. (2001), Phys. Rev. B 63, 224522.

[28] Schirber, J.E. (1970), Cryogenics 10, 418.

[29] See, for example, Sadewasser, S., Schilling, J.S., and Hermann, A.M. (2000), Phys. Rev. B 62, 9155 .

[30] Lorenz, B., Meng, R.L., and Chu, C.W., preprint cond-mat/0104303.

[31] Deemyad, S., Schilling, J.S., Jorgensen, J.D., and Hinks D.G. (2001), Physica C 361, 227.

[32] Tissen, V.G., Nefedova, M.V., Kolesnikov, N.N., and Kulakov, M.P., preprint condmat/0105475.

[33] Osborn, R., Goremychkin, E.A., Kolesnikov, A.I., Hinks, D.G. (2001), Phys. Rev. Lett. 87, 017005.

[34] An J.M., and Pickett, W.E. (2001), Phys. Rev. Lett. 86, 4366.

[35] Kong, Y., Dolgov, O.V., Jepsen, O., and Andersen, O.K. (2001), Phys. Rev. B 64, 020501.

[36] Medvedeva, N.I., Ivanovskii, A.L., Medvedeva, J.E., Freeman, A.J. (2001), Phys. Rev. B 64, 020502.

[37] Kortus, J., Mazin, I.I., Belashchenko, K.D., Antropov, V.P., Boyer, L.L. (2001), Phys. Rev. Lett. 86, 4656.

[38] Neaton, J.B., and Perali, A., preprint cond-mat/0104098.

[39] Medvedera, N.I., Ivanovskii, A.L., Medvedeva, J.E., Freeman, A.J., and Novokov, D.L., (preprint cond-mat/0104346).

[40] Roundy, D., Choi, H.J., Sun, H., Louie, S.G., Cohen, M.L., post deadline session on $\mathrm{MgB}_{2}$, Monday evening, March 12, 2001, APS March Meeting in Seattle, Washington.

[41] Chen, X.J., Zhang, H., and Habermeier, H.-U., preprint.

[42] Loa, I., and Syassen, K. (2001), Solid State Commun. 118, 279.

[43] Bud'ko, S.L., Lapertot, G., Petrovic, C., Cunningham, C.E., Anderson, N., Canfield, P. C. (2001), Phys. Rev. Lett. 86, 1877. 
[44] Hinks, D., Claus, G.H., Jorgensen, J. (2001), Nature 411, 457.

[45] Tanigaki, K., Ebbesen, T.W., Saito, S., Mizuki, J., Tsai, J.S., Kubo, Y. and Kuroshima, S. (1991), Nature 352, 222.

[46] Schilling, J.S., Diederichs, J., and Gangopadhyay, A.K. (1997), in K.M. Kadish and R.S. Ruoff (ed.), Fullerenes: Recent Advances in the Chemistry and Physics of Fullerenes and Related Materials, Vol. IV, Electrochemical Society, p. 980.

[47] Diederichs, J., Gangopadhyay, A.K., and Schilling, J.S. (1996), Phys. Rev. B 54, R9662.

[48] Diederichs, J., Schilling, J.S., Herwig, K.W., and Yelon, W.B. (1997), J. Phys. Chem. Solids 58, 123.

[49] Schön, J.H., Kloc, Ch., and Batlogg, B. (2000), Nature 406, 704.

[50] Schön, J. H., Kloc, Ch., and Batlogg B. (2000), Nature 408, 549.

[51] Bednorz, J.G., and Müller, K.A. (1986), Z. Physik 64, 189.

[52] Schilling, A., Cantoni, M., Guo, J.D., and Ott, H.R. (1993), Physica C 178, 183.

[53] Gao, L., Xue, Y.Y., Chen, F., Xiong, Q., Meng, R.L., Ramirez, D., Chu, C.W., Eggert, J.H., and Mao, H.-k. (1994), Phys. Rev. B 50, 4260.

[54] Ran, C.Y., Lin, W., Wu, Y.S., Yin, B., and Tang, D.S. (1998), Low Temp. Phys. 24, 230.

[55] Djurek, D., Medunić, Z., Tonejc, A., and Paljević, M. (2001), Physica C 351, 78.

[56] Presland, M.R., Tallon, J.L., Buckley, R.G., Liu, R.S., and Flower, N.D. (1991), Physica $C$ 176, 95.

[57] Jorgensen, J.D., Hinks, D.G., Chmaisem, O., Argyriou, D.N., Mitchell, J.F., and Dabrowski, B. (1996) in Lecture Notes in Physics-Proceedings of the first Polish-US Conference on High Temperature Superconductivity, 11-15 September 1995, WroclawDuszniki Zdr., Poland, Springer-Verlag.

[58] Takahashi, H. and Mori, N. (1995), in A.V. Narlikar (ed.), Studies of High Temperature Superconductors, Vol. 16, Nova Science, New York, p. 1.

[59] Schirber, J.E., Venturini, E.L., Morosin, B., Ginley, D.S. (1989), Physica C 162, 745.

[60] Klehe, A.-K., Schilling, J.S., Wagner, J.L., and Hinks, D.G. (1994), Physica C 223, 313. 
[61] Klehe, A.-K., Looney, C., Schilling, J.S., Takahashi, H., Mori, N., Shimakawa, Y., Kubo, Y., Manako, T., Doyle, S., and Hermann, A.M. (1996), Physica C 257, 105.

[62] Sadewasser, S., Schilling, J.S., Paulikas, A.P., and Veal, B.W. (2000), Phys. Rev. B 61, 741 .

[63] Sadewasser, S., Schilling, J.S., Hermann, A.M. (2000), Phys. Rev. B 62, 9155.

[64] Allgeier, C., Schilling, J.S., Ku, H.C., Klavins, P., and Shelton, R.N. (1987), Solid State Commun. 64, 227.

[65] Allgeier, C., Heise, J., Reith, W., Schilling, J.S., and Andres, K. (1989), Physica C 157, 293.

[66] Meingast, C., Wolf, T., Kläser, M., and Müller-Vogt, G. (1996), J. Low Temp. Phys. 105, 1391.

[67] Wijngaarden, R.J., Jover, D.T., and Griessen, R. (1999), Physica B 265, 128. 
This figure "Fig2new.png" is available in "png" format from: http://arxiv.org/ps/cond-mat/0110267v1 
This figure "LatticeParameterGraphic.png" is available in "png" format from: http://arxiv.org/ps/cond-mat/0110267v1 
This figure "T-P-mcmillan.png" is available in "png" format from: http://arxiv.org/ps/cond-mat/0110267v1 
This figure "Tcn3.png" is available in "png" format from: http://arxiv.org/ps/cond-mat/0110267v1 
This figure "fullerenes3.png" is available in "png" format from: http://arxiv.org/ps/cond-mat/0110267v1 